\title{
Connecting solar radiance variability to the solar dynamo with the virial theorem
}

\section{Oskar Steiner}

Kiepenheuer-Institut für Sonnenphysik, Schöneckstrasse 6, D-79104 Freiburg, Germany email: steiner@kis.uni-freiburg.de

\begin{abstract}
The variability of solar radiance over a solar cycle is thought to be a delicate balance between the radiative deficit of sunspots and the extra contribution of plage and network regions. Although the net effect is tiny, it must imply structural and thermal changes in the Sun or in partial layers of it as an unavoidable consequence of the virial theorem. Using the virial theorem for continua including the magnetic field it is shown, how solar radiance variability might be connected to a deeply seated flux-tube dynamo and how this connection is established on a hydrodynamical time-scale.
\end{abstract}

\section{What can the virial theorem tell us?}

Assuming that the global solar luminosity varies approximately with solar irradiance, then the virial theorem unavoidable implies structural and thermal changes of the Sun or partial layers of it on that same time scale. What kind of changes may it be?

A general form of the virial theorem for continua including the magnetic field, is given by Chandrasekhar \& Fermi (1953):

$$
\frac{1}{2} \frac{\mathrm{d}^{2} J}{\mathrm{~d} t^{2}}=2 K+\Omega+M+3 \int_{\mathcal{R}} P \mathrm{~d} V+S,
$$

where $J$ is the moment of inertia, $K$ the kinetic energy of mass motion, $\Omega$ the gravitational binding energy of the star, $M$ the magnetic energy, $P$ the gas pressure, and $S$ a surface integral over the boundary $\partial \mathcal{R}$ of the region $\mathcal{R}$ occupied by the star. This surface integral vanishes if the magnetic field at $\partial \mathcal{R}$ is either parallel to $\partial \mathcal{R}$ or sufficiently weak, and if $P \approx 0$ which, at the solar surface, is both approximately fulfilled.

The virial theorem is an integral equation, allowing to extract information on global quantities only. We may try to confine the region of interest, $\mathcal{R}$, within two boundaries, an outer one near the solar surface with $\mathbf{B} \approx P \approx 0$ and an inner one near the overshoot layer at the bottom of the convection zone but already within the radiation zone with $\mathbf{B}=0$ and $P=$ const., where we assume that the solar core shall not be influenced by the solar cycle at all. Then, $S=$ const. and $\mathcal{R}$ encompasses the convection zone for which we may estimate the variation of the various virials over the solar cycle.

\section{Estimates of the various virials}

We estimate the change of each virial term in Eq. (1.1) over a solar cycle. One solar cycle generates a magnetic flux of $\Phi \approx 10^{16} \mathrm{~Wb}$ (Galloway \& Weiss, 1981). If this flux resides in the overshoot layer in the form of a flux sheet with a strength of $10 \mathrm{~T}$, it has a thickness of $d \approx 10^{6} \mathrm{~m}$ and a total magnetic energy of

$$
M \approx 10^{32} \mathrm{~J},
$$

where the latter number is independent of the filling factor (Rempel, 2001). 
This magnetic energy is mainly generated in the last stage of the intensification process, say from 1 to $10 \mathrm{~T}$. The initial magnetic layer with flux density $1 \mathrm{~T}$ has a thickness of $10^{7} \mathrm{~m}$ for to harbour $10^{16} \mathrm{~Wb}$ magnetic flux, within a volume of $3 \times 10^{25} \mathrm{~m}^{3}$. Within this volume, the available kinetic energy of convective motion is $K_{\text {conv }} \approx 0.1 \times M$. Also, from mixing-length theory, the convective energy transport is $F_{\mathrm{c}} \propto v_{\mathrm{c}}^{2}$, from where it follows that $v_{\mathrm{c}}^{2}$ may not vary more than $10^{-3}$ over the solar cycle.

In the layer where magnetic intensification from 1 to $10 \mathrm{~T}$ takes place, the total available kinetic energy from differential rotation under the constraint of constant angular momentum is $K_{\text {diff }}<0.1 \times M$ (Rempel, 2001), while we assume that there exists no external torque. Note, however, that for the entire convection zone $K_{\text {rot }} \approx 500 \times M$. Differential rotation in the magnetic layer would have to be very efficiently replenished from layers atop for feeding the energy required for the field intensification (Moreno Insertis, Caligari \& Schüssler, 1995). Therefore, we hypothetically set

$$
K(t)=\frac{1}{2} \int_{\mathcal{R}} \rho \mathbf{v}^{2} \mathrm{~d} V=\text { const. }
$$

Thus, kinetic energy is assumed not to be a major source for field intensification although differential rotation is certainly an important agent for generating the $(<1 \mathrm{~T})$ toroidal field in the first place. One would have thought that the torsional oscillation was a manifestation of Lorentz forces acting on the Plasma. Yet Spruit (2003) comes to the conclusion that it is due to a geostrophic flow driven by temperature variations at the surface that are themselves a consequence of solar radiance variability.

In that same volume, the potentially available momentum is

$$
\mathrm{d}^{2} J / \mathrm{d} t^{2} \approx J / \tau_{\text {cyc }}^{2} \approx 10^{-3} \times M
$$

The equivalent variation in thermal energy over a solar cycle that is exclusively due to the solar luminosity variation, $\delta L \approx 0.001 \cdot L_{\odot}$, is

$$
\Delta U_{\mathrm{rad}} \approx 10^{32} \mathrm{~J} \text {. }
$$

This coincidence of equal magnetic and thermal energy change over a solar cycle was noticed by Schüssler (1996). The idea here is to obtain a connection with the help of the virial theorem.

Considering variations over a solar cycle, three significant virials of the full virial equation remain:

$$
\Omega+M+2 U=0 .
$$

A possible scenario that relates the three terms is provided by the flux-tube dynamo that operates in the overshoot layer at the bottom of the convection zone.

\section{Flux intensification and entropy transport by large-scale flux tubes}

In the flux-tube dynamo, magnetic field is generated in a 11 year cycle near the bottom of the convection zone from where strands of flux undergo undular instability (Ferriz Mas \& Schüssler, 1995) when magnetic loops start rising into the convection zone (Fig. 1).

As these flux tubes further rise through the convection zone, the gas pressure decreases less rapidly in the adiabatic flux-tube atmosphere, $p_{\mathrm{i}}$, than it does in the superadiabatically stratified environment, $p_{\mathrm{e}}$ (Fig. 2). At the critical height, $z_{\text {crit }}=z_{\text {exp }}$, the magnetic pressure must vanish and the flux tube "explodes" into a cloud of weak field (Moreno Insertis, Caligari \& Schüssler, 1995, Rempel \& Schüssler, 2001). As a consequence of the flux-tube explosion, mass gets "sucked" from the flux tube buried in the overshoot layer 


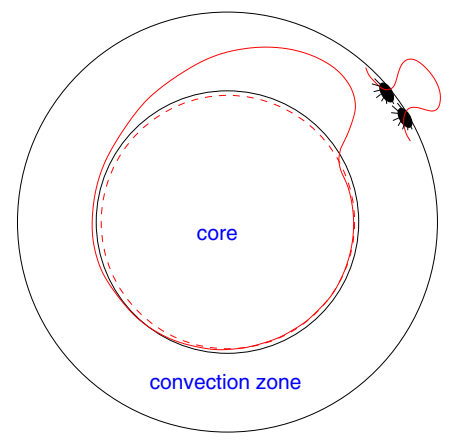

Figure 1. Magnetic flux tube at the bottom of the convection zone (dashed circle), rising through the convection zone to the surface (solid red curve), where it forms a sunspot pair.

at the bottom of the convection zone, which leads to an increase in flux density and thus in magnetic energy.

Another consequence is that entropy-rich plasma gets transported from the bottom, and injected into the upper layers of the convection zone on a hydrodynamical time-scale of approximately one month. Rempel (2001) estimates, that the energy carried by this flux over one solar cycle is $\Delta U \approx 1 \times M$.

The process of flux-tube rising and flux-tube explosion can be understood as a magnetoconvective mixing-length transport similar to but more efficient than the regular hydrodynamic one as this "magnetoconvective mixing length" can be a large fraction of the convection-zone depth. By this process, the convection zone finds a way of more efficiently transport energy across it, limited by the amount of available magnetic flux.

\section{The solar cycle in terms of the virial theorem}

Starting with a weak toroidal field of $B \approx 1 \mathrm{~T}$, this field gets amplified to $10 \mathrm{~T}$ by the process of "flux-tube" explosions, increasing the field energy by

$$
\Delta M=M_{2}-M_{1}=|M| \approx 10^{32} \mathrm{~J},
$$

so that, according to the virial theorem, Eq. (2.5),

$$
\Delta U=U_{2}-U_{1}=-\frac{1}{2}(\Delta \Omega+|M|) .
$$
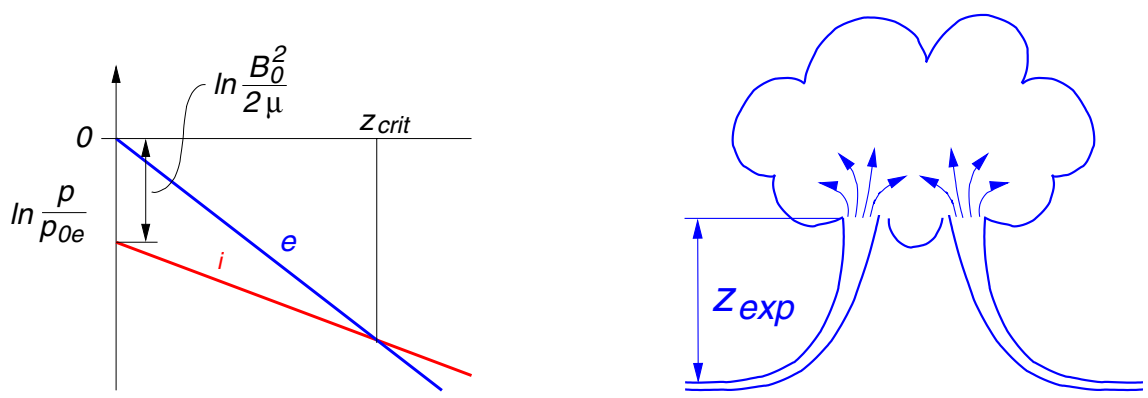

Figure 2. Left: Gas pressure decreases less rapidly in the adiabatic flux-tube atmosphere, $p_{i}$, than it does in the superadiabatic environment, $p_{e}$. Right: At the critical height, $z_{\text {crit }}=z_{\exp }$, the magnetic pressure must vanish and the flux tube "explodes" into a cloud of weak field. 

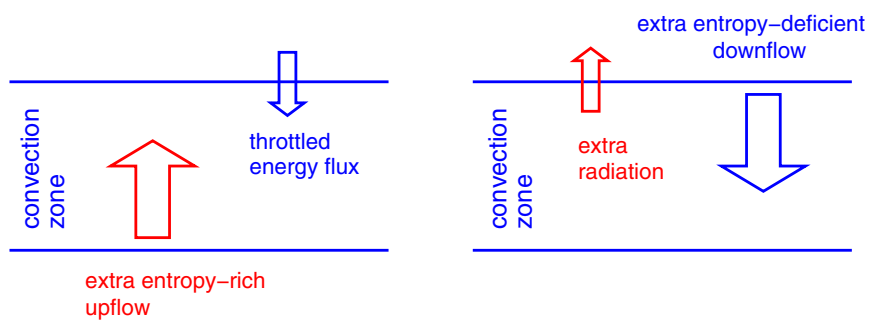

Figure 3. The thermodynamic cycle associated with the magnetic solar cycle.

Except for the rising loops, the flux tubes stay in mechanical equilibrium during the intensification process, which hints that $\Delta \Omega \approx 0$, but a rigorous proof is still missing. If so, Eq.(4.2) leaves an internal energy deficiency of another $-10^{32} \mathrm{~J}$, which must be gained by a reduction in radiation loss from the Sun in fulfillment of the total energy equation. A reduction of radiation loss could be caused by a reduction of the superadiabaticity, $\delta=\nabla-\nabla_{\mathrm{ad}}$, which throttles the convective energy transport, $F_{\mathrm{c}} \propto \delta . \delta$ in turn is reduced by the excess of entropy injected into the convection zone by the magnetoconvective process of flux-tube rise and flux-tube explosion.

In the second half of the cycle $\Delta M=-|M| \approx-10^{32} \mathrm{~J}$ because all the magnetic field gets ejected or annihilated, clearing stage for the next solar cycle with opposite magnetic sign. This is the time of magnetic activity at the solar surface. Again, assuming $\Delta \Omega \approx 0$ (or $\Delta \Omega<0$ for to obtain $\Delta U>0$ ), Eq. (2.5) leads us to

$$
\Delta U=-\frac{1}{2}(\Delta \Omega-|M|) \approx+10^{32} \mathrm{~J}>0,
$$

leaving an internal energy excess of $10^{32} \mathrm{~J}$, which must be lost by an excess of radiation from the Sun.

Excessive radiation loss is caused by radiative channeling in magnetic elements of network and plage regions. In these regions entropy deficient material is transported in downflow plumes back to the depth of the convection zone. Accordingly, the solar cycle is accompanied by a thermodynamic cycle illustrated in Fig. 3. The magnetoconvective transport of entropy-rich material into the convection zone with associated reduction of convective energy transport (1st stage) and the formation of plage and network faculae with associated transport of entropy-deficient material back into the convection zone (2nd stage), all processes work on a hydrodynamic time-scale, so that the response for structural changes caused by the dynamo process takes place on the hydrodynamical, not the Kelvin-Helmholtz time-scale.

\section{References}

Chandrasekhar, S. \& Fermi, E. 1953 ApJ 118, 116.

Ferriz Mas, A. \& Schüssler, M. 1995 Geophys. Astrophys. Fluid Dyn. 81, 233

Fröhlich, C., and Lean, J. 1998 In New eyes to see inside the Sun and stars (ed. F.-L. Deubner), IAU Symp. 185, Kluwer, Dordrecht

Galloway, D.J. \& Weiss, N.O. 1981 ApJ 243, 945.

Moreno Insertis, F., Caligari, P. \& Schüssler M. 1995 ApJ 452, 894.

Rempel, M. 2001 Struktur und Ursprung starker Magnetfelder am Boden der solaren Konvektionszone PhD-Thesis, Georg-August-Universität Göttingen, Duehrkohp \& Radicke, Göttingen

Rempel, M. \& Schüssler, M. 2001 ApJ 552, L171.

Schüssler, M. 1996 In Solar and Astrophysical Magnetohydrodynamics (ed. K.C. Tsiganos), NATO ASI Series, vol. 486, p. 17, Kluwer, Dordrecht

Spruit, H. C. 2003 Solar Phys. 213, 1-21. 